\title{
Existence of superposition solutions for pulse propagation in nonlinear resonant media
}

\author{
P. K. Panigrahi * 1,2 and G. S. Agarwal ${ }^{\dagger} 1$ \\ 1 Physical Research Laboratory, Navrangpura, Ahmedabad-380 009, India \\ 2 School of Physics, University of Hyderabad, Hyderabad-500 046, India
}

\begin{abstract}
Existence of self-similar, superposed pulse-train solutions of the nonlinear, coupled Maxwell-Schrödinger equations, with the frequencies controlled by the oscillator strengths of the transitions, is established. Some of these excitations are specific to the resonant media, with energy levels in the configurations of $\Lambda$ and $N$ and arise because of the interference effects of cnoidal waves, as evidenced from some recently discovered identities involving the Jacobian elliptic functions. Interestingly, these excitations also admit a dual interpretation as single pulse-trains, with widely different amplitudes, which can lead to substantially different field intensities and population densities in different atomic levels.
\end{abstract}

PACS number(s): 42.50.Md, 42.50.Hz, 42.65.Tg

*prasanta@prl.res.in

†gsa@prl.res.in 


\section{INTRODUCTION}

The generation of shape preserving excitations in nonlinear media has been the subject of extensive research in diverse areas of physics, ranging from hydrodynamics [1], particle physics [2] to quantum optics [3-5] and optical communications [6]. Starting from the explanation of the solitary waves in shallow water [7], these solutions of the nonlinear wave equations have found ample experimental verifications. In optical context, the nonlinear nature of the coupled Maxwell- Schrödinger equations [8], describing the interaction of classical radiation with matter in a resonant media, has naturally generated tremendous interest in the study of the pulse [3,9-13] and pulse-train solutions [14-17]. Recently the continuous pulse-train soliton solutions have been observed experimentally for the two-level system [18]. For a detailed overview, the interested readers are referred to Refs.( $[19,20])$, apart from the above references.

The common factor that governs the existence of the self-similar excitations in various nonlinear systems is the critical balance between nonlinearity and dispersion [21]. This severely restricts the set of solutions, in most of the cases, to combinations of the Jacobian elliptic functions [22] and also leads to definite relationships between the amplitudes, widths and velocities of these modes. The periodic elliptic functions, characterized by the modulus parameter $m$ and smoothly interpolating between hyperbolic secant and tangent to periodic cosine and sine functions, for $m$ values one and zero respectively, describe localized pulses for $m=1$ and pulse-trains for $0 \leq m<1$.

In this light, the recent findings of a number of identities involving the superposition of elliptic functions [23-25] and their application to various nonlinear systems [26] makes it extremely interesting to find the implications of the same for the multi-level resonant media. A number of phenomena, like self [27] and electromagnetically induced transparencies [28], pulse sharpening [29], pulse cloning and dragging [30], just to name a few, are ascribable to the existence of shape-preserving excitations in resonant media. A great deal of attention, both analytical and numerical, has been paid to the cases in which the resonant atoms are 
asymptotically in the ground or excited state. Pulse train solutions, characteristic to excited media, have been studied, for two-level $[14,15]$ and for three and five-level [16] configurations. Well-known techniques for generating solutions e.g., inverse scattering methods [21,31,32] and Bäcklund transformations [33] have also been employed for higher level atomic media, albeit under restrictive conditions on the propagation constants and other parameters of these systems. Further progress, in the understanding of the dynamics of the inhomogeneously broadened three level system, has recently been achieved by the derivation of an area theorem [34].

In this paper, we first show the existence of novel pulse train solutions, specific to the $\Lambda$ and $N$-type, nonlinear, resonant media, taking recourse to the above mentioned identities, involving elliptic functions. Interestingly, these solutions can either be viewed as a linear superposition of cnoidal waves or as single cnoidal waves, with widely different, modulus dependent amplitudes for different pulse trains. We find that, for some of these exact solutions, the modulus parameter is controlled by the oscillator strengths of the atomic transitions, as compared to other pulse train solutions found so far, where $m$ appears as a free parameter. Interference effects, originating from the superposition nature of these solutions, lead to substantially different field intensities and population densities in different atomic levels. We then point out the existence of pulse train solutions in the four-level case, similar to the ones found in other multi-level systems.

\section{SUPERPOSED PULSE TRAINS IN $\Lambda$ SYSTEMS}

We start with the three-level $\Lambda$ system, because of its wide applicability and then proceed to the four-level case, with the energy levels in the configuration of $N$, from which the other lower ones will follow under limiting conditions. Shape preserving solutions, in the form of superposition of a pair of different cnoidal waves (like a superposition of $\mathrm{sn}(\mathrm{x}, \mathrm{m})$ and

$\mathrm{cn}(\mathrm{x}, \mathrm{m}))$, have been obtained earlier by Hioe and Grobe [16], for the three and five level systems. These exact solitary waves can have a variety of shapes, because of interference. 
The solutions to be discussed here are superpositions of an odd number of cnoidal waves of a given type (no combinations involving, say both $\mathrm{sn}(\mathrm{x}, \mathrm{m})$ and $\mathrm{cn}(\mathrm{x}, \mathrm{m})$ ), with appropriately displaced arguments. As will be seen later in the text, these superposed solutions can have widely different amplitudes. This can be understood from the fact that, both $\mathrm{cn}(\mathrm{x}, \mathrm{m})$ and $\mathrm{sn}(\mathrm{x}, \mathrm{m})$ functions take values ranging from -1 to 1 , whereas the $\mathrm{dn}(\mathrm{x}, \mathrm{m})$ function has only positive values. Hence, the superposed solutions involving $\mathrm{cn}(\mathrm{x}, \mathrm{m})$ and $\mathrm{sn}(\mathrm{x}, \mathrm{m})$ functions can have a much smaller amplitude as compared to the one involving $\operatorname{dn}(\mathrm{x}, \mathrm{m})$ function, whose amplitude can be substantially higher.

For the $\Lambda$ system consisting of the ground state $i$, excited state $e$ and the intermediate state $f$, we assume the fields to have slowly varying pulse envelopes: $\overrightarrow{\mathrm{E}_{\alpha}}(\mathrm{z}, \mathrm{t})=$ $\overrightarrow{\mathcal{E}_{\alpha}}(\mathrm{z}, \mathrm{t}) \mathrm{e}^{-\mathrm{i}\left(\omega_{\alpha} \mathrm{t}-\mathrm{k}_{\alpha} \mathrm{z}\right)}+$ c.c. . In the rotating wave approximation, $\overrightarrow{\mathcal{E}}_{e}$, and $\overrightarrow{\mathcal{E}}_{f}$ act on transitions $e i$ and $e f$, respectively. The Rabi frequencies, $\Omega_{\alpha}=2 \frac{\overrightarrow{d_{\alpha}} \overrightarrow{\mathcal{E}_{\alpha}}}{\hbar}$, with $\overrightarrow{d_{\alpha}}$ being the dipole matrix element of the $\alpha$-th transition, are also slowly varying functions of space and time. We assume, for simplicity that, all the fields are resonant with their respective transitions.

In slowly varying envelope approximations, the resonant coupled Maxwell-Schrödinger equations are

$$
\begin{aligned}
i \frac{\partial}{\partial \tau} C_{e} & =-\frac{\Omega_{f}}{2} C_{f}-\frac{\Omega_{e}}{2} C_{i} \\
i \frac{\partial}{\partial \tau} C_{i} & =-\frac{\Omega_{e}^{\star}}{2} C_{e} \\
i \frac{\partial}{\partial \tau} C_{f} & =-\frac{\Omega_{f}^{\star}}{2} C_{e} \\
\frac{\partial}{\partial \zeta} \Omega_{f} & =i \mu_{f} C_{e} C_{f}^{\star} \\
\frac{\partial}{\partial \zeta} \Omega_{e} & =i \mu_{e} C_{e} C_{i}^{\star} .
\end{aligned}
$$

In above, we have neglected the relaxation terms, since the pulse widths are taken to be smaller than the relaxation times. $C_{\alpha}(\alpha=e, i, f)$ represents the probability amplitude of finding the atom in the state $\mid \alpha>$.

The parameter $\mu_{\alpha}$ is given by $\mu_{\alpha}=4 \pi \mathcal{N} \frac{\left|d_{\alpha}\right|^{2} \omega_{\alpha}}{\hbar c}=\frac{2 \pi e^{2} \mathcal{N} f_{\alpha}}{m c}$ where $\mathcal{N}$ is the density of the 
atoms and $f_{\alpha}$ being the oscillator strength for the transition of frequency $\omega_{\alpha}$. The atomic system is assumed to be continuously distributed in a non-dispersive host medium. The coordinate $\tau=t-\frac{z}{c}$ measures time relative to the pulse center and $\zeta=z$.

We look for self-similar solutions of Eqs. (1) and (2) i.e., solutions depending upon a single variable, $X=(q \zeta-\Gamma \tau)$. Here, $\Gamma^{-1}$ is the pulse duration and, as will be seen later, $q$ and $\Gamma$ will be related via the pulse velocity and another parameter, characterizing the solutions. The superposed pulse-train solutions, to be discussed here, appear in both $\Lambda$ and $N$ type media and can be made to satisfy a wide range of initial conditions, like no occupancy of the desired atomic levels to partial occupation of all the levels. It should be pointed out that partial occupation of levels necessarily implies initially induced coherence in the atomic system. We note that, the pulse train solutions of $\Lambda, V$ and other odd-level systems [16], consisting of a matched pair of elliptic functions, do not satisfy the nonlinear equations of the $N$ system.

It can be shown that, the following ansatz solutions:

$$
C_{i}=\tilde{S}, C_{f}=b_{f} \tilde{C}, C_{e}=b_{e} \tilde{D}, \Omega_{f}=A_{f} \tilde{S}, \Omega_{e}=A_{e} \tilde{C}
$$

satisfy the Maxwell-Schrödinger equations, provided the functions $\tilde{C}, \tilde{S}$ and $\tilde{D}$ are as defined below and the constant coefficients $b_{f}, b_{e}$ and $A_{e}, A_{f}$ are appropriately related. $\tilde{C}, \tilde{S}$ and $\tilde{D}$ are linear superpositions of the Jacobi elliptic functions $\mathrm{cn}(\mathrm{X}, \mathrm{m}), \operatorname{sn}(\mathrm{X}, \mathrm{m})$ and $\mathrm{dn}(\mathrm{X}, \mathrm{m})$, respectively: $\tilde{S}=\sum_{i=1}^{p} \operatorname{sn}\left[\mathrm{X}+\frac{4(\mathrm{i}-1) \mathrm{K}(\mathrm{m})}{\mathrm{p}}, \mathrm{m}\right]$ and $\tilde{C}$ and $\tilde{D}$ are analogously defined. Here, $p$ is an odd integer, $K(m)$ is the complete elliptic integral of the first kind and $m$ is the modulus parameter. The above solutions are possible for odd integral values of $p$, since the cross terms in the right hand side of Eqs.(1) and (2) are cancelled because of the identities of the type [23],

$$
\begin{aligned}
& \tilde{s_{1}}\left(\tilde{d}_{2}+\tilde{d_{3}}\right)+\text { cyclic permutations }=0, \\
& \tilde{c_{1}}\left(\tilde{s_{2}}+\tilde{s_{3}}\right)+\text { cyclic permutations }=0, \\
& \tilde{c_{1}}\left(\tilde{d}_{2}+\tilde{d}_{3}\right)+\text { cyclic permutations }=0 .
\end{aligned}
$$


Here, $\tilde{s_{1}} \equiv \operatorname{sn}(\mathrm{X}, \mathrm{m}), \tilde{s_{2}} \equiv \operatorname{sn}\left(\mathrm{X}+\frac{4 \mathrm{~K}(\mathrm{~m})}{\mathrm{p}}, \mathrm{m}\right)$ and $\tilde{s_{3}} \equiv \operatorname{sn}\left(\mathrm{X}+\frac{8 \mathrm{~K}(\mathrm{~m})}{\mathrm{p}}, \mathrm{m}\right)$ and other functions are similarly defined. Although, for definiteness, henceforth we consider only the $p=3$ case, the consistency conditions on the parameters, written below, are identical for all values of $p$. Introducing an additional parameter $\mu=2 q \Gamma m$, for future convenience, one finds,

$$
\begin{aligned}
\left|A_{e}\right|^{2} & =4 \Gamma^{2} m \frac{\mu_{e}}{\mu} \\
\left|A_{f}\right|^{2} & =4 \Gamma^{2} m\left[\frac{\mu_{e}}{\mu}-1\right], \\
b_{e} & =\frac{i q A_{e}}{\mu_{e}} \\
b_{f} & =-\frac{A_{f}^{\star}}{A_{e}^{\star}}
\end{aligned}
$$

and also the constraint, $\mu_{e}=\mu_{f}$. The positive definite character of the pulse intensity, requires that $\mu_{e} \geq \mu$.

The superposed character of the solutions, for higher values of $p$, leads to significant differences between different pulse-train and electron amplitudes, as will be explicated later. It also manifests in the conservation of probabilities for the electrons. For the $p=3$ case, $\sum_{\alpha}\left|C_{\alpha}\right|^{2}=1$ leads to,

$$
\frac{\mu_{e}-\mu}{\mu_{e}}=\frac{\frac{1}{m}-1+\frac{4}{m}\left(\tilde{q}^{2}+\tilde{q}\right)}{\frac{1}{m}-1+\frac{2}{m}\left[(\tilde{q}+1)^{2}-\frac{m}{(\tilde{q}+1)^{2}}\right]} .
$$

Here, $\tilde{q}=\operatorname{dn}\left(\frac{2}{3} \mathrm{~K}(\mathrm{~m}), \mathrm{m}\right)$ and satisfies,

$$
\tilde{q}^{4}+2 \tilde{q}^{3}-2(1-m) \tilde{q}-(1-m)=0
$$

The values of $\tilde{q}$ range from one to zero continuously, when $m$ varies between zero and one. It can be checked that, when, $m=1$, the conservation law is identically satisfied, making the above equation vacuous; in this case one obtains the pulse solutions of the $\Lambda$ system. For $m=0$, right hand side takes value one, which is ruled out on physical grounds. For other values of $m$, the above equation can be numerically solved to obtain the range of values of $\mu$ for a given $\mu_{e}$. It should be noted that, since $\mu_{e} \geq \mu$, the variable $\frac{\mu_{e}-\mu}{\mu_{e}}$ takes values 
between zero and one. It is found that for the superposed solutions to exist, the condition $0.59 \lesssim \frac{\mu_{e}-\mu}{\mu_{e}}<1$ should be satisfied. As depicted in Fig.1,

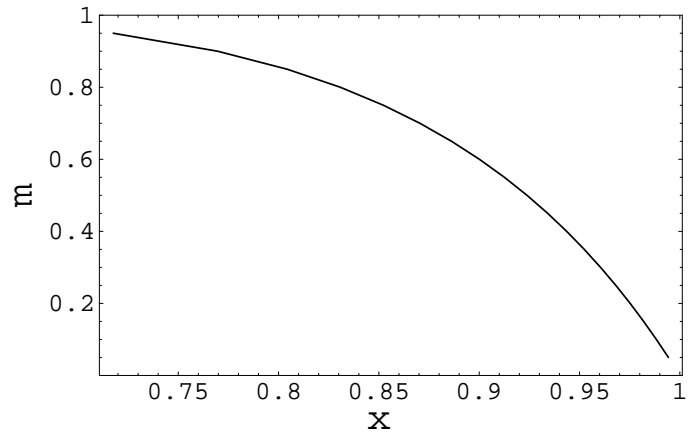

FIG. 1. Graph of the modulus parameter $m$ versus $x=\frac{\mu_{e}-\mu}{\mu_{e}}$.

the modulus parameter $m$ can lie between one and zero, for the above values of $\frac{\mu_{e}-\mu}{\mu_{e}}$. Hence the choice of $m(0<m<1)$ determines $\mu$ through Eq.(7), which in turn yields $q \Gamma$, through $\mu=2 q \Gamma m$. Hence the solutions are characterized by the two free parameters $m$ and $\Gamma$. It can be easily seen that $\tilde{D}$ has a much higher amplitude as compared to $\tilde{C}$ and $\tilde{S}$; hence the $\mid e>$ state has a higher population. The superposition nature of the solutions enables one to have these widely differing amplitudes. It can be checked that exchange of $\tilde{C}$ and $\tilde{D}$ also leads to allowed solutions. In that case the $\mid f>$ state population can be made large as compared to $\mid i>$ and $\mid e>$ states.

For the purpose of comparison, we consider the non-superposed $p=1$ case. Taking $C_{i}=b_{i} \operatorname{sn}(\mathrm{X}, \mathrm{m})$ and replacing $\tilde{D}$ and $\tilde{C}$ by corresponding cnoidal waves one finds, after appropriately choosing the fields, that, the parameter relationships as obtained in Eqs. $(5,6)$ are unchanged. However, the probability conservation now yields substantially different result:

$$
\frac{1}{\left|b_{i}\right|^{2}}=\frac{\mu}{\mu_{e}}\left(\frac{1}{m}-1\right)+1 \text {. }
$$

It is clear from the above expression that, for, $b_{i}=1$, no pulse train solutions are possible, as $m=1$, for this case. Since $\mu / \mu_{e}$ lies between zero and one, it follows from Eq.(9) that, $\left|b_{i}\right|^{2}$ takes values ranging from $m$ and 1 . It should be pointed that, like the previous 
case, $0<m<1$. One is then finally left with three free parameters, $\Gamma, m$ and $b_{i}$, in their appropriate range of values. It is possible to obtain another solution by exchanging $\mathrm{dn}(\mathrm{X}, \mathrm{m})$ with $\mathrm{cn}(\mathrm{X}, \mathrm{m})$, in the above ansatz. This choice leads to the constraint,

$$
\frac{1}{\left|b_{i}\right|^{2}}=\left(1-\frac{\mu}{\mu_{e}}\right) \frac{1}{m} \text {. }
$$

\section{PULSE TRAIN SOLUTIONS OF THE $N$ SYSTEM}

In case of the $N$ system, one needs to consider seven coupled equations, due to the presence of an additional energy state $\mid v>$, in between the states $\mid e>$ and $\mid f>$, of the $\Lambda$ system. Here, one has the additional parameter, $\mu_{v}=\frac{2 \pi e^{2} \mathcal{N} f_{v}}{m c}, f_{v}$ being the corresponding oscillator strength. These nonlinear equations have been recently found to possess propagating pulse solutions [13]. Introducing, the additional electron amplitude $C_{v}=b_{v} \tilde{D}$ and the Rabi frequency $\Omega_{v}=A_{v} \tilde{C}$ in the ansatz solutions, we only write below, the new and modified parametric relations, as compared to the $\Lambda$ system:

$$
\begin{aligned}
2 q \Gamma m & =\mu_{v}, \\
\left|A_{v}\right|^{2} & =4 \Gamma^{2} m\left[1-\frac{\mu_{e}}{\mu_{f}}\right], \\
\left|A_{e}\right|^{2} & =4 \Gamma^{2} m \frac{\mu_{e}^{2}}{\mu_{f} \mu_{v}}, \\
b_{v} & =\frac{i q A_{v}}{\mu_{v}} \\
b_{f} & =-\frac{A_{f}^{\star}}{A_{e}^{\star}} \frac{\mu_{e}}{\mu_{f}} .
\end{aligned}
$$

Note that here, $\mu_{v}$ determines $2 q \Gamma m$, unlike the $\Lambda$ system. The positive definite character of the pulse intensities now requires the inequalities,

$$
\mu_{f} \geq \mu_{e} \geq \mu_{v} .
$$

In the present case, the conservation of probability yields, 


$$
\frac{\mu_{e}-\mu_{v}}{\mu_{f}}=\frac{\frac{1}{m}-1+\frac{4}{m}\left(\tilde{q}^{2}+\tilde{q}\right)}{\frac{1}{m}-1+\frac{2}{m}\left[(\tilde{q}+1)^{2}-\frac{m}{(\tilde{q}+1)^{2}}\right]} .
$$

This leads to the constraint on the oscillator strengths: $0.59 \lesssim \frac{\mu_{e}-\mu_{v}}{\mu_{f}}<1$, for $0<m<1$. It is worth noting that, as compared to $\Lambda$-system, in the present case the oscillator strengths determine modulus parameter $m$.

The uniform group velocity, as determined by our solutions is given by,

$$
\frac{1}{v}=\frac{1}{c}+\frac{\mu_{v}}{2 \Gamma^{2} m}
$$

As the width of the pulse train becomes shorter ( $\Gamma$ gets larger $)$, the group velocity approaches the background medium velocity; however, for smaller values of $m$, the pulse train velocity can be significantly smaller than the medium velocity. We have checked that a different superposition type solution, e.g., $C_{i}=\tilde{S}$ and exchange of $\tilde{C}$ and $\tilde{D}$ for other fields, do not alter the above conclusions. However, the population of different levels, as well as the field amplitudes can be substantially different, from the earlier case. It is straightforward to see, from Eqs.(11) and (15), that unlike the $\Lambda$-system, the $N$-system is characterized by one free parameter $\Gamma$.

It is worth noting that, for the two level case, corresponding to $\mu_{e}=\mu_{v}=\mu_{f}$, as well as for the $V$ system, for which $\mu_{e}=\mu_{v}$, the constraint equation originating from the probability conservation yields,

$$
m=1+4\left(\tilde{q}^{2}+\tilde{q}\right)
$$

Since $0 \leq m \leq 1$ and $\tilde{q}>0$, it is easy to see that, the above equation can not be satisfied. Hence these type of excitations are exclusive to $\Lambda$ and $N$ type systems.

A better understanding of the above solutions can be provided by a dual interpretation of these excitations, through a set of generalized Landen transformations, found recently. For the $p=3$ case, the transformation formula yields,

$$
\alpha \operatorname{dn}(\mathrm{x}, \tilde{\mathrm{m}})=\left[\operatorname{dn}(\alpha \mathrm{x}, \mathrm{m})+\operatorname{dn}\left(\alpha \mathrm{x}+\frac{4 \mathrm{~K}(\mathrm{~m})}{3}, \mathrm{~m}\right)+\operatorname{dn}\left(\alpha \mathrm{x}+\frac{8 \mathrm{~K}(\mathrm{~m})}{3}, \mathrm{~m}\right)\right]
$$


where $\alpha=\left[\operatorname{dn}(0, \mathrm{~m})+\operatorname{dn}\left(\frac{4 \mathrm{~K}(\mathrm{~m})}{3}, \mathrm{~m}\right)+\operatorname{dn}\left(\frac{8 \mathrm{~K}(\mathrm{~m})}{3}, \mathrm{~m}\right)\right]$ and

$$
\tilde{m}=m \frac{(1-\tilde{q})^{2}}{(1+\tilde{q})^{2}(1+2 \tilde{q})^{2}},
$$

with $\tilde{q}=\operatorname{dn}\left(\frac{2}{3} \mathrm{~K}(\mathrm{~m}), \mathrm{m}\right) \tilde{q}$. For the $\operatorname{sn}(\mathrm{x}, \tilde{\mathrm{m}})$ and $\mathrm{cn}(\mathrm{x}, \tilde{\mathrm{m}})$ functions, the formulae have same widths in right hand side as in $\operatorname{dn}(\mathrm{x}, \tilde{\mathrm{m}})$; however, the amplitude $\alpha$ is replaced by $\beta$, given by

$$
\beta=\left[\operatorname{cn}(0, \mathrm{~m})+\operatorname{cn}\left(\frac{4 \mathrm{~K}(\mathrm{~m})}{3}, \mathrm{~m}\right)+\operatorname{cn}\left(\frac{8 \mathrm{~K}(\mathrm{~m})}{3}, \mathrm{~m}\right)\right]
$$

The facts that, $\operatorname{dn}(\mathrm{x}, \mathrm{m})$ takes only positive values and $\mathrm{cn}(\mathrm{x}, \mathrm{m})$ have values ranging from +1 to -1 , can be seen to be the reason behind the large value of $\alpha$ as compared to that of $\beta$. Hence, the superposed cnoidal waves can have widely varying amplitudes, which leads to significant differences in atomic level occupations.

\section{PURE CNOIDAL SOLUTIONS FOR THE $N$ SYSTEM}

Since the four-level system has not been systematically analyzed for pulse train solutions, we would like to point out that, like the two-level case, pure cnoidal waves, with comparable electron and field amplitudes, can also be obtained as solutions to the $N$ system. As has been mentioned earlier, matched pair type pulse train solutions of the odd-level atomic systems [16] are not solutions of the four-level system. It can be seen that, the following ansatz solutions:

$$
C_{i}=b_{i} \operatorname{sn}(\mathrm{X}, \mathrm{m}), \mathrm{C}_{\mathrm{f}}=\mathrm{b}_{\mathrm{f}} \mathrm{dn}(\mathrm{X}, \mathrm{m}), \mathrm{C}_{\mathrm{e}}=\mathrm{b}_{\mathrm{e}} \operatorname{cn}(\mathrm{X}, \mathrm{m}), \mathrm{C}_{\mathrm{v}}=\mathrm{b}_{\mathrm{v}} \mathrm{dn}(\mathrm{X}, \mathrm{m})
$$

and

$$
\Omega_{f}=A_{f} \operatorname{sn}(\mathrm{X}, \mathrm{m}), \Omega_{\mathrm{e}}=\mathrm{A}_{\mathrm{e}} \operatorname{dn}(\mathrm{X}, \mathrm{m}), \Omega_{\mathrm{v}}=\mathrm{A}_{\mathrm{v}} \operatorname{dn}(\mathrm{X}, \mathrm{m})
$$

satisfy the Maxwell-Schrödinger equations, provided $\left|b_{i}\right|^{2}<1$ and the constant coefficients $b_{i}, b_{f}, b_{e}, b_{v}$ and $A_{e}, A_{f}, A_{v}$ are related. The consistency conditions on all the parameters are now modified: 


$$
\begin{aligned}
\frac{2 q \Gamma m}{\left|b_{i}\right|^{2}} & =\mu_{v}, \\
\left|A_{v}\right|^{2} & =4 \Gamma^{2}\left[1-\frac{\mu_{e}}{\mu_{f}}\right], \\
\left|A_{f}\right|^{2} & =4 \Gamma^{2} m\left[\frac{\mu_{e}}{\mu_{v}}-1\right], \\
\left|A_{e}\right|^{2} & =4 \Gamma^{2} \frac{\mu_{e}^{2}}{\mu_{f} \mu_{v}}, \\
b_{e} & =\frac{i \mu_{v} A_{e} b_{i}}{2 \mu_{e} \Gamma}, \\
b_{v} & =\frac{i A_{v} b_{i}}{2 \Gamma}, \\
b_{f} & =-\frac{A_{f}^{\star}}{A_{e}^{\star}} \frac{\mu_{e} b_{i}}{m \mu_{f}}, \\
\frac{1}{\left|b_{i}\right|^{2}} & =\left[1+\frac{1-m}{m}\left(\frac{\mu_{e}-\mu_{v}}{\mu_{f}}\right)\right] .
\end{aligned}
$$

For a given ground state occupancy, the modulus parameter $m$ is determined by Eq.(23). The velocity is now modified and depends upon $\left|b_{i}\right|^{2}$ :

$$
\frac{1}{v}=\frac{1}{c}+\frac{\mu_{v}\left|b_{i}\right|^{2}}{2 \Gamma^{2} m} \text {. }
$$

For the purpose of considering limiting cases of the $N$ system, we observe that, when the modulus parameter $m$ equals one, the cnoidal functions go over to hyperbolic ones, thereby yielding localized pulse solutions. The pulse trains are obtained, when $0<m<1$, the $m=0$ value being ruled out in the present case. Since $\frac{\mu_{e}-\mu_{v}}{\mu_{f}}$ is positive, it is clear from Eq. that, for the $N$ system, $\left|b_{i}\right|^{2}$ must be less than one, for the latter type of solutions. However, for the two level case, where $\mu_{e}=\mu_{v}=\mu_{f}$, the above restriction does not apply. In that case, $b_{i}=1$ and one obtains the cnoidal solutions, similar to a set of solutions, obtained by Crisp and Arecchi et al. in Ref. [14], in an inhomogeneous media. Exchanging dn(X, m) with $\mathrm{cn}(\mathrm{X}, \mathrm{m})$ in the above ansatz, leads to $\left|b_{i}\right|^{2}=m$; in this case one obtains the solutions, analogous to another set of solutions given in Ref. [14], under inhomogeneous conditions. This is also clear from the analysis of the $\Lambda$ system, which is obtained from the $N$ system under the condition, $\mu_{e}=\mu_{f}$, with $\mu=\mu_{v}$. The above point also indicates that for the $\Lambda$ system, obtained as a limiting case, $\left|b_{i}\right|^{2}<1$, in order for the pulse train solution to exist. One obtains localized pulse solutions for $\left|b_{i}\right|^{2}=1$, since in that case $m=1$. For the $V$ 
system, for which $\mu_{e}=\mu_{v}$, the conclusions are similar to the two-level case. A comparison with Ref. [16] shows that the above solutions form a subclass of the matched pair solutions, for the $\Lambda$ and $V$ system. This result is expected since matched pair type superposition solutions are not possible for the $N$ system.

For the purpose of better appreciation of the relative amplitude variations of the superposed solutions, as compared to the pure cnoidal ones, Fig.2 depicts two of the pulse trains with their cnoidal constituents. The widely different amplitudes of the superposed $\operatorname{dn}(X, m)$ and $\operatorname{sn}(X, m)$ type cnoidal waves and their respective constituents are clearly visible.

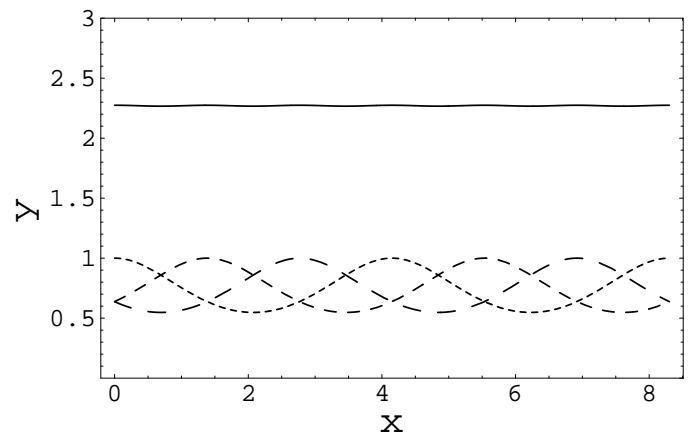

FIG. 2a. Plot depicting the superposed $\tilde{D}(X, m)$ (solid curve) and its three $\operatorname{dn}(X, m)$ constituents (dotted curves), for $m=0.7$.

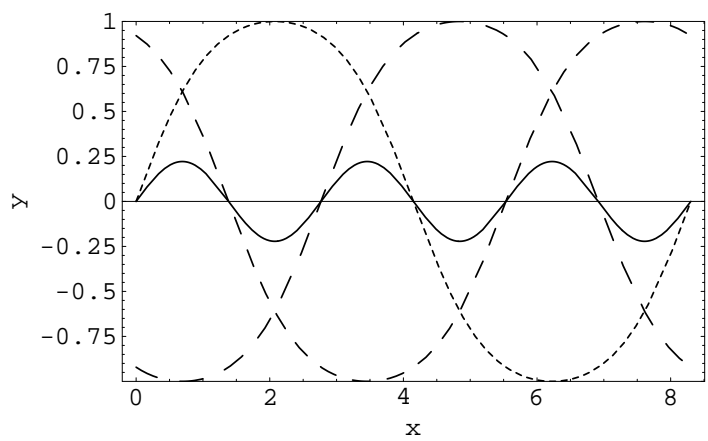

FIG. 2b. Plot depicting the superposed $\tilde{S}(X, m)$ (solid curve) and its three cnoidal constituents (dotted curve), for $m=0.7$.

Fig.3 shows, the superposed solutions with their non-superposed counterparts, clearly bringing out their differences. 


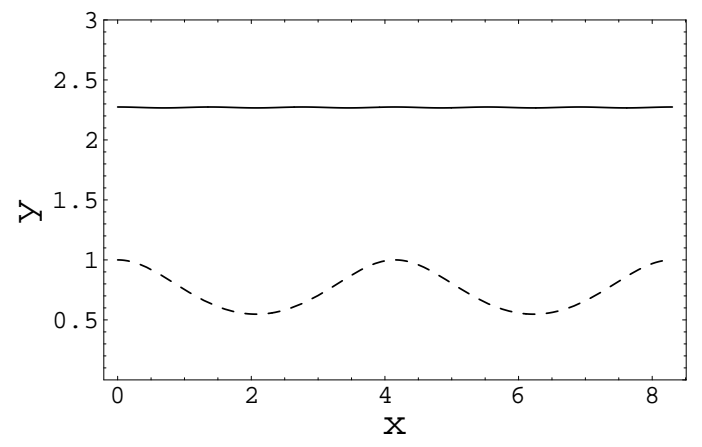

FIG. 3a. Plots depicting the superposed solution $\tilde{D}(X, m)$ (solid curve) of Eq.(3) and its non-superposed counterpart (dotted curve) in the same units, for $m=0.7$. These plots represent the electron amplitudes in the state $\mid e>$ of the $\Lambda$ system.

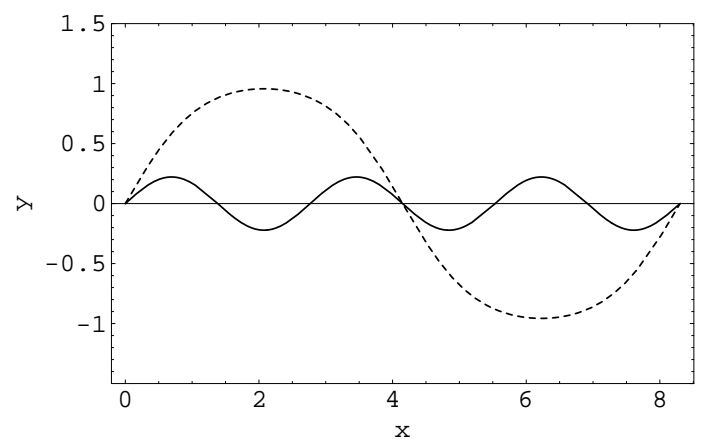

FIG. 3b. Plots depicting the superposed $\operatorname{sn}(\mathrm{X}, \mathrm{m})$ solution (solid curve) of Eq.(3) and its pure cnoidal counterpart (dotted curve), for $m=0.7$ and $b_{i}=0.9869$. With $b_{i}=1$ these plots represent the field strengths $\Omega_{f}$ of the $\Lambda$ system, in the same units.

Fig.4 reveals the same for different parameter values. One sees that, for $m=0.3$ the amplitude of superposed $\operatorname{sn}(X, m)$ solution attains a very small value. It is extremely interesting to observe that, the excited state probability density can be made very small and other densities enhanced, through these novel solutions, which is not possible through pure cnoidal waves. It is clear that, this desirable feature of the pulse trains owes its origin to the superposition nature of the solutions. 


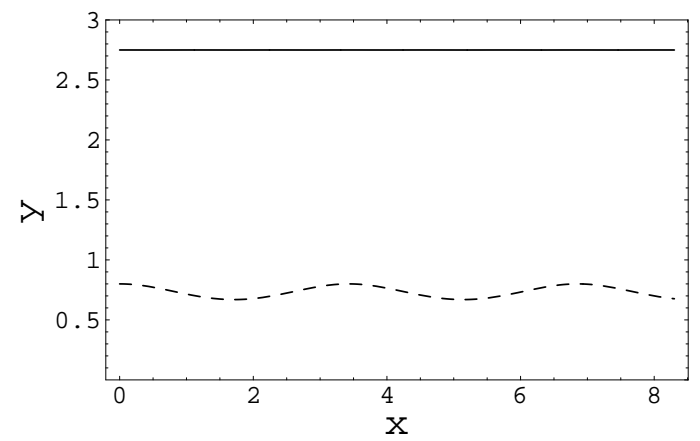

FIG. 4a. Plots depicting the superposed $\tilde{D}(X, m)$ (solid curve) and its non-superposed counterpart (dotted curve) in the same units, for $m=0.3$.

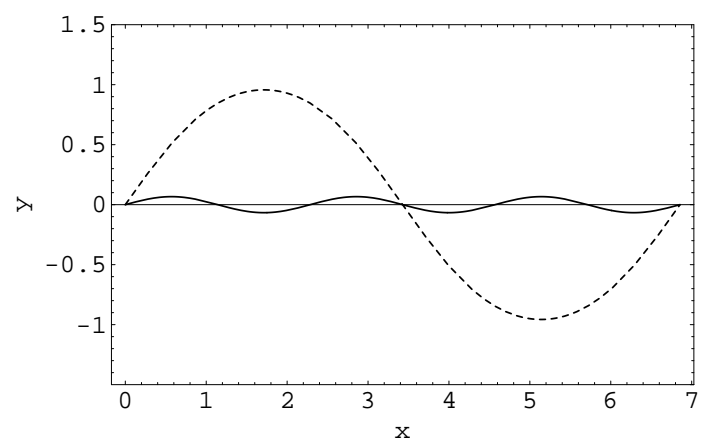

FIG. 4b. Plots depicting the superposed $\operatorname{sn}(\mathrm{X}, \mathrm{m})$ solution (solid curve) and its pure cnoidal counterpart (dotted curve), for $m=0.3$ and $b_{i}=0.9568$.

It is natural to enquire the effect of detunings on the above solutions and to find if the previous methods of incorporating detuning (particularly the case $\Delta_{e}=\Delta_{f}$ in the $\Lambda$ system [12]) would also work for deriving the pulse train solutions.

\section{CONCLUSIONS}

In summary, we have found that delicate interference phenomena involving cnoidal waves give rise to certain novel shape-preserving excitations, exclusive to $\Lambda$ and $N$ type multi-level atomic media. It was also observed that matched pair-type self-similar solutions, possible in odd-level systems, do not occur in the even-level $N$ system, although a variety of simple 
pulse-train solutions are possible in the latter case. Interestingly, the superposition nature of the above mentioned excitations of the nonlinear media, makes it possible for different atomic levels to have widely different population densities and different pulse trains possess significantly differing amplitudes, features not present in simple pulse-train solutions. For the $N$-system, the frequencies of the propagating pulses, which govern the spatio-temporal behavior of the atomic population densities, are not arbitrary, as have been the case so far, with the pulse-train solutions of various systems. The modulus parameter $m$, which appears in the velocities and also controls the period $K(m)$ is determined by the propagation constants. In light of the potential usefulness of the multi-level systems, starting from information storage $[35,36]$ to quantum computation [37], we hope that, some of these desirable features of the excitations may find practical applications. It is interesting to observe that, presence of additional non-linearities leads to chirping of the pulse train of the two-level systems [38]. The effect of similar non-linearities on the superposed and non-superposed pulse trains solutions, for the three and four-level systems is worth further investigation.

One of the authors (PKP) acknowledges useful discussions with Prof. A. Khare. 


\section{REFERENCES}

[1] C.B. Whitham, Linear and Nonlinear Waves (Wiley, New York, 1974).

[2] R. Jackiw, Rev. Mod. Phys. 49, 681 (1977).

[3] S.L. McCall and E.L. Hahn, Phys. Rev. Lett. 18, 908 (1967).

[4] H. Steudel, Physica 6D, 155 (1983).

[5] R. Grobe, F.T. Hioe and J.H. Eberly, Phys. Rev. Lett. 73, 3183 (1994).

[6] G.P. Agrawal, Nonlinear Fiber Optics (Academic Press, New York, 1989); A. Hasegawa, Optical Solitons in Fibers (Springer-Verlag, Berlin, 1989).

[7] D.J. Korteweg and G. de Vries, Phil. Mag.(5) 39, 422 (1895).

[8] L. Allen and J.H. Eberly, Optical Resonances and Two-Level Atoms (Dover, New York, 1987).

[9] G.L. Lamb, Jr., Phys. Lett. A 25, 181 (1967).

[10] G.L. Lamb, Jr., Rev. Mod. Phys. 43, 99 (1971).

[11] M.J. Konopnicki and J.H. Eberly, Phys. Rev. A 24, 2567 (1981).

[12] A. Rahman and J.H. Eberly, Phys. Rev. A 58, R805 (1998).

[13] G.S. Agarwal and J.H. Eberly, Phys. Rev. A 61, 13404 (1999).

[14] J.H. Eberly, Phys. Rev. Lett. 22, 760 (1969); F.T. Arechi, V. DeGiorgio and S.G. Someda, Phys. Lett. A 27, 588 (1968); M.D. Crisp, Phys. Rev. Lett. 22, 820 (1969).

[15] T.W. Barnard, Phys. Rev. A 7, 373 (1973).

[16] F.T. Hioe and R. Grobe, Phys. Rev. Lett. 73, 2559 (1994).

[17] M.A. Newbold and G.J. Salamo, Phys. Rev. Lett. 42, 887 (1979).

[18] J.L. Shultz and G.J. Salamo Phys. Rev. Lett. 78, 855 (1997). 
[19] A.I. Maimistov, A.M. Basharov, S.O. Elyutin and Yu.M. Sklyarov, Phys. Rep. C 191, $1(1990)$.

[20] A.V. Andreev, JETP 86, 412 (1998).

[21] A. Das, Integrable Models (World Scientific, Singapore, 1989); P.G. Drazin and R.S. Johnson, Solitons: An Introduction (Cambridge Univ. Press, 1989).

[22] H. Hancock, Theory of Elliptic Functions (Dover, New York, 1958); M. Abramowitz and I. Stegun, Handbook of Mathematical Functions (NBS, US Government Printing Office, 1964).

[23] A. Khare and U. Sukhatme, Jour. Math. Phys. 43, 3798 (2002).

[24] A. Khare and U. Sukhatme, math-ph/0204054.

[25] A. Khare, A. Lakshminarayan and U. Sukhatme, math-ph/0207019.

[26] A. Khare and U. Sukhatme, Phys. Rev. Lett. 88, 244101 (2002).

[27] S.L. McCall and E.L. Hahn, Phys. Rev. 183, 457 (1969).

[28] S.E. Harris, Phys. Today 50(7), 36 (1997).

[29] L.A. Bol'shov, N.N. Elkin, V.V. Likhanskii and M.I. Persiantsev, Sov. Phys. JETP 61(1), 27 (1985).

[30] G. Vemuri, G.S. Agarwal, Phys. Rev. Lett. 79, 3889 (1997).

[31] L.A. Bol'shov, N.N. Elkin, V.V. Likhanskii and P.A. Napartovich, Sov. J. Quantum Electron. 12, 941 (1982); L.A. Bol'shov and V.V. Likhanskii, ibid. 15, 889 (1985).

[32] A.M. Basharov and A.I. Maimistov, Sov. Phys. JETP 67, 2426 (1988).

[33] H. Steudel, J. Mod. Opt. 35, 693 (1988); Q.-H. Park and H.J. Shin, Phys. Rev. A 57, 4643 (1998). 
[34] J.H. Eberly and V.V. Kozlov, Phys. Rev. Lett. 88, 243604 (2002).

[35] C. Liu, Z. Dutton, C.H. Behroozi, and L.V. Hau, Nature (London) 409, 490 (2001); D.E. Phillips, A. Fleischhauer, A. Mair, R. L. Walsworth and M.D. Lukin, Phys. Rev. Lett. 86, 783 (2001).

[36] T.N. Dey and G.S. Agarwal, quant-ph/0209099.

[37] W. Teich and G. Mahler, Complexity, Entropy and the Physics of Information, SFI studies in Sciences of Complexity, Vol. VIII, W. Zurek (ed.), Addison-Wesley, Reading, MA, 289 (1990).

[38] L. Matulic, P.W. Milonni and J.H. Eberly, Opt. Commun. 4, 181 (1971); L. Matulic and J.H. Eberly, Phys. Rev. A 6, 822, 1258 (1972). 